\title{
Consulta de alta resolución de digestivo en Andalucía: ¿qué es y cómo debe funcionar?
}

\author{
J. M. Herrerías Gutiérrez, F. Argüelles Arias, L. Martín Herrera ${ }^{1}$, J. M. Montero Domínguez ${ }^{2}$, \\ J. de Dios Vega ${ }^{3}$, R. Martín-Vivaldi Martínez ${ }^{4}$, A. Domínguez Macías ${ }^{5}, J_{\text {. Maldonado Eloy-García }}^{6}$, \\ A. M. Sánchez Cantos ${ }^{7}$, M. Romero Gómez ${ }^{8}$ y J. L. Márquez Galán
}

Servicio de Aparato Digestivo. Hospital Virgen de la Macarena. Sevilla. ${ }^{1}$ Hospital Puerta del Mar. Cádiz. ${ }^{2}$ Hospital Puerto Real. Cádiz. ${ }^{3}$ Hospital Reina Sofía. Córdoba. ${ }^{4}$ Hospital Virgen de las Nieves. Granada. ${ }^{5}$ Hospital Juan Ramón Jiménez. Huelva. ${ }^{6}$ Hospital Regional Carlos Haya. ${ }^{7}$ Hospital Costa del Sol. Marbella, Málaga. ${ }^{8}$ Hospital Nuestra Señora del Valme. Sevilla. ${ }^{9}$ Hospital Universitario Virgen del Rocío. Sevilla

\section{RESUMEN}

Introducción: la idea actual de nuestro sistema sanitario es que la asistencia sea ambulatoria y que se utilice la hospitalización cuando sea precisa. En este sentido es de destacar el desarrollo de la consulta única o de alta resolución. Por ello, se ha realizado una encuesta entre varios hospitales andaluces con la idea de definir y determinar qué aspectos son necesarios para poder desarrollar esta consulta.

Material y métodos: la encuesta ha sido contestada por 10 hospitales andaluces. Se trata de un estudio prospectivo descriptivo de las respuestas contestadas por los distintos hospitales. Las preguntas son 27 en las que se reflexiona sobre la existencia de la consulta y la infraestructura para desarrollarla: cuántos pacientes se ven, dónde se pasa esta consulta, de dónde provienen los pacientes, los criterios para derivar los pacientes a esta consulta, las condiciones en las que acude el enfermo, si se realiza ecografía de abdomen, si se dispone de sistema informático integrado hospitalario, enfermera, en cuántas visitas se emite un diagnóstico del paciente $\mathrm{y}$, por último, se pregunta si se cree que es necesaria esta consulta y por qué.

Resultados: de los 10 hospitales 5 tienen consulta de alta resolución, aunque todos consideran que la deberían tener. El número de pacientes atendidos debe ser 10 y en el propio hospital. Existen diferencias en considerar si los pacientes deben provenir desde Urgencias o desde el médico de cabecera. Parece lógico pensar que sólo se deben derivar pacientes cuya patología pueda ser diagnosticada mediante ecografía y/o endoscopia. El paciente debería acudir a la consulta en ayunas y con analítica del médico de cabecera, para así poder realizarles la ecografía. La consulta debe constar de sistema informático y de una enfermera propia. Según los encuestados este tipo de consultas es muy útil en nuestro actual sistema, porque permite mayor colaboración entre Atención Primaria y el especialista, y consigue una orientación rápida de la patología del paciente actuando de filtro adecuado para el resto de las consultas.

\begin{abstract}
Introduction: the present concept in our healthcare system is that medical care should be given on an outpatient basis with hospitalization occurring only when essential. We therefore put forth the development of the "all in one" outpatient office or "high resolution" outpatient clinic. For such purpose we administered a questionnaire to various Andalusian hospitals to define and determine those aspects necessary in the development of the aforementioned outpatient office.

Materials and methods: the questionnaire was filled out by 10 Andalusian hospitals. This is a prospective-descriptive study of responses from all 10 participating hospitals. The 27 questions inquired on the existence of such an outpatient office and the infrastructure needed to develop this service: How many patients are seen, where is it physically located, where do patients come from, criteria for assigning patients to this medical office, condition of incoming patients, whether ultrasound scans are performed, whether an integrated hospital computer system exists, nursing staff, how many visits are required before coming to a diagnosis, and finally whether this type of outpatient office is needed, and if so, why.

Results: of all 10 hospitals, 5 of them had this type of clinic. All of them considered this type of outpatient service essential. The number of patients treated should be "10", in the hospital itself. There are differences as to whether patients should come from the emergency room or a primary care physician. It seems logical to assume that only patients who can be diagnosed via ultrasounds or endoscopy should be chosen. To allow an ultrasonogram the patient should visit the outpatient office in a state of "fasting" and with standard blood counts from the primary care physician.

The outpatient clinic should have a computer system and its own nurse. According to participating hospitals this type of outpatient visits is very useful in our present healthcare system, as it allows higher levels of collaboration between Primary Care and the specialist; it also provides a rapid orientation regarding patient pathology, and acts as a "filter" for the rest of the healthcare system.
\end{abstract}


Conclusiones: la consulta debe ser atendida por un facultativo especialista de área (FEA) con conocimientos y experiencia en ecografía, endoscopia digestiva y en el manejo de programas informáticos a nivel de usuario. Hoy en día puede ser considerada una modalidad de consulta resolutiva y eficaz en nuestro sistema sanitario.

Palabras clave: Consulta de alta resolución. Consultas de digestivo. Funcionamiento de consultas de digestivo.
Conclusions: the outpatient office should be tended to by an attending specialist in the field (FEA) with knowledge and experience in ultrasounds and gastrointestinal endoscopy, as well as user competency with the required computer programs. In our present-day system this can be considered a modality of high-resolution outpatient services and a model of efficiency.

Key words: High resolution outpatient clinic. Gastroenterology outpatient offices.

Herrerías Gutiérrez JM, Argüelles Arias F, Martín Herrera L, Montero Domínguez JM, de Dios Vega J, Martín-Vivaldi Martínez R, Domínguez Macías A, Maldonado Eloy-García J, Sánchez Cantos AM, Romero Gómez M, Márquez Galán JL. Consulta de alta resolución de digestivo en Andalucía: ¿qué es y cómo debe funcionar? Rev Esp Enferm Dig 2008; 100: 5-10.

\section{INTRODUCCIÓN}

La idea actual de nuestro sistema sanitario es que la asistencia sanitaria sea ambulatoria de forma que se utilice la hospitalización cuando sea precisa y durante el menor tiempo necesario. La consulta externa aporta ventajas al paciente y al sistema sanitario: mayor comodidad para el paciente y menor coste para el sistema sanitario.

En este sentido es de destacar el desarrollo de alternativas como la consulta única o de alta resolución. El uso de actividad asistencial en consultas externas debe ser considerado hoy en día imprescindible y necesario para el buen funcionamiento de nuestro sistema sanitario de tal forma que la mayor parte de la atención al paciente sea llevado en las consultas. Sólo se ingresarán aquellos pacientes que necesiten una atención hospitalaria.

Se trata de inducir un uso lo más eficiente del hospital disminuyendo los ingresos hospitalarios y tratando de diagnosticar y tratar al paciente de forma ambulatoria. Todo lo anterior entronca con el concepto de hospitalización inapropiada, para lo cual se utilizaron diversos instrumentos de identificación, de los que el más conocido y utilizado es el Appropriateness Evaluation Protocol. En un estudio realizado en España, Aplicación del Appropriateness Evaluation Protocol (AEP) en un hospital de agudos, se trataba de medir la reproductibilidad del AEP y cuantificar el grado de inadecuación hospitalaria en un hospital de agudos y determinar las posibles causas que lo justifican. Se encontró un 15\% (IC 95\%: 11-19) de inadecuación en ingresos con un 20\% (IC 95\%: 16-24) de estancias consideradas inadecuadas. La principal causa de inadecuación de ingresos fue la admisión innecesaria por tratarse de cuidados que podrían haberse realizado de manera ambulatoria. En el hospital, los factores causantes de inadecuación fueron: el tratamiento conservador de los médicos y la disponibilidad de esos cuidados a nivel ambulatorio. Así los autores concluyen que el AEP, como instrumento específico para cuantificar la inadecuación hospitalaria e identificar sus causas, se perfila como una herramienta útil en la actualidad para establecer programas de intervención dirigidos a reducir el uso hospitalario inadecuado.

Las consultas externas de digestivo sufren en la actualidad una masificación importante debido a la alta prevalencia de patología digestiva y la necesidad de realizar pruebas complementarias (endoscopias) que también presentan lista de espera. No existen datos extraídos de organismos oficiales de la proporción de pacientes que consultan por un motivo u otro a una consulta especializada de digestivo, aunque de manera general se puede decir que la patología funcional (dispepsia y síndrome de intestino irritable) supone un $40 \%$ del total. Varios diagnósticos son los más emitidos por el especialista en digestivo: enfermedad por reflujo gastresofágico, dispepsia funcional, síndrome de intestino irritable, hemorroides, lesiones inducidas por el ácido gástrico, litiasis biliar, etc.

En la mayoría de los casos estos diagnósticos se alcanzan con una endoscopia, una ecografía abdominal y una analítica.

Por tanto, parece que organizar una consulta de alta resolución de digestivo (CARE) puede tener cabida en nuestro sistema sanitario.

\section{OBJETIVOS}

Es por esto, y para orientar cómo está funcionando en nuestra comunidad este nuevo método de consulta, que se ha realizado una encuesta entre varios hospitales andaluces con la idea de definir, configurar y determinar qué aspectos son necesarios para poder desarrollar esta consulta de acto único. 


\section{MATERIAL Y MÉTODOS}

La encuesta ha sido contestada por 10 hospitales:

- Hospital Virgen Macarena de Sevilla.

- H. Virgen del Rocío de Sevilla.

$-\mathrm{H}$. Virgen de Valme de Sevilla.

- H. Costa del Sol de Marbella.

- H. Puerta del Mar de Cádiz.

- H. Juan Ramón Jiménez de Huelva.

- H. Reina Sofía de Córdoba.

- Hospital Carlos Haya de Málaga.

- Hospital Puerto Real de Cádiz.

- Hospital Virgen de las Nieves de Granada.

\section{ESTUDIO ESTADÍSTICO}

Se trata de un estudio prospectivo en el que se realiza un estudio estadístico meramente descriptivo de las respuestas contestadas por los distintos hospitales.

\section{Encuesta}

Cada hospital ha respondido al siguiente cuestionario:

1. ¿Existe en su centro una CARE?

$\square$ Sí.

$\square$ No.

2. ¿Cree que debería existir?

$\square$ Sí.

$\square$ No.

3. En caso de que exista, ¿cuántos pacientes se ven?

$\square$ Menos de 10.

$\square$ Entre 11 y 15 .

$\square$ Entre 16 y 20.

$\square$ Más de 20.

4. ¿Cuántos cree que se deberían ver?

$\square$ Menos de 10 .

$\square$ Entre 11 y 15 .

$\square$ Entre 16 y 20.

$\square$ Más de 20.

5. ¿Dónde se pasa esta consulta?

$\square$ En el centro de especialidades médicas (CEEM).

$\square$ En el hospital.

6. ¿Dónde cree que se debería pasar?

$\square$ En el centro de especialidades médicas (CEEM).

$\square$ En el hospital.

7. ¿De dónde provienen los pacientes?

$\square$ Derivados por el médico de cabecera.

$\square$ Derivados por otros especialistas no digestivos o por los digestivos del hospital. $\square$ Desde urgencias.

$\square$ Todos los casos.

8. ¿De dónde cree que deberían provenir?

$\square$ Derivados por el médico de cabecera.

$\square$ Derivados por otros especialistas no digestivos o por los digestivos del hospital.

$\square$ Desde urgencias.

$\square$ Todos los casos.

9. ¿Existe algún criterio para derivar los pacientes a esta consulta?

$\square$ Son derivados los más urgentes o con cuadros agudos.

$\square$ Son derivados al azar sin seguir ningún criterio.

$\square$ Son derivados los pacientes con sospecha de patología funcional.

10. ¿Cree que debería existir algún criterio para derivar los pacientes a esta consulta?

$\square$ Los más urgentes o con cuadros agudos.

$\square \mathrm{Al}$ azar sin seguir ningún criterio.

$\square$ Los pacientes con sospecha de patología funcional.

$\square$ Los pacientes que presenten una patología concreta.

11. ¿En qué condiciones acude el enfermo a la consulta?

$\square$ En ayunas.

$\square$ Con una analítica del médico de cabecera.

$\square$ Ambas condiciones.

$\square$ Sin ninguna preparación especial.

12. ¿En qué condiciones debería acudir el enfermo a la consulta?

$\square$ En ayunas.

$\square$ Con una analítica del médico de cabecera.

$\square$ Ambas condiciones.

$\square$ Sin ninguna preparación especial.

13. En la misma consulta, ¿se realiza ecografía de abdomen al paciente?

$\square$ Sí.

$\neg$ No.

14. ¿Cree que se debería realizar?

$\square$ Sí.

$\square$ No.

15. ¿Dispone de sistema informático integrado hospitalario?

$\square$ Sí.

$\square$ No.

16. ¿Cree que debería disponer de sistema informático integrado hospitalario?

$\square$ Sí.

$\square$ No. 
17. ¿Dispone de una enfermera para esa consulta en exclusiva?

$\square$ Sí.

$\square$ No.

18. ¿Cree que debería disponer de una enfermera para esa consulta en exclusiva?

$\square$ Sí.

$\square$ No.

19. En general, ¿en cuántas visitas se emite un diagnóstico del paciente?

$\square$ En 1.

$\square$ En 2.

$\square$ En 3 o más.

20. En general, ¿en cuántas visitas se debería emitir un diagnóstico del paciente?

$\square$ En 1.

$\square$ En 2.

$\square$ En 3 o más.

21. ¿Quién vuelve a citar el enfermo?

$\square$ La enfermera.

$\square$ El médico.

$\square$ El administrativo.

22. ¿Quién debería volver a citar el enfermo?

$\square$ La enfermera.

$\square$ El médico.

$\square$ El administrativo.

23. En caso de no poderse dar de alta al paciente por presentar una patología que precise revisiones por digestivo...

$\square$ Se deriva a otra consulta.

$\square$ Se sigue en esta consulta CARE.

24. ¿Qué piensa que debería hacerse en el anterior caso expuesto?

$\square$ Se debe derivar a otra consulta.

$\square$ Se debe seguir en esta consulta CARE.

25. ¿Cree que es útil esta consulta?

$\square$ Sí.

$\square$ No

26. En caso afirmativo, ¿por qué?

27. En caso negativo, ¿por qué?

\section{RESULTADOS}

- Pregunta 1: ¿Existe en su centro una CARE?

- 5 hospitales sí la tienen y 5 no.
- Pregunta 2: ¿Cree que debería existir?

- Todos responden que sí.

-Pregunta 3: En caso que exista, ¿cuántos pacientes se ven?

- 4 hospitales entre 16 y 20, uno más de 10 .

-Pregunta 4: ¿Cuántos cree que se deberían ver?

- Todos los hospitales creen que deben verse menos de 10 .

- Pregunta 5: ¿Dónde se pasa esta consulta?

- 4 en el hospital, 1 en el CEEM.

- Pregunta 6: ¿Dónde cree que se debería pasar?

- 8 en el hospital, 2 en el CEEM.

- Pregunta 7: ¿De dónde provienen los pacientes?

- 2 contestan desde urgencias y 3 todos los casos.

- Pregunta 8: ¿De dónde cree que deberían provenir?

- 3 contestan que: todos los casos anteriores.

- 7 contestan desde urgencias y derivados por el médico de cabecera.

- Pregunta 9: ¿Existe algún criterio para derivar los pacientes a esta consulta?

- 2 contestan que son derivados al azar sin seguir ningún criterio.

- Otros 2 contestan que son derivados los más urgentes o con cuadros agudos.

- 1 contesta sí pero sin especificar.

- Pregunta 10: ¿Cree que debería existir algún criterio para derivar los pacientes a esta consulta?

- 2 contestan que los más urgentes o con cuadros agudos y 2 contestan que sólo los pacientes que presenten una patología concreta.

- 1 contesta que sólo aquellos cuya patología se pueda resolver mediante eco y endoscopia.

-Pregunta 11: ¿En qué condiciones acude el enfermo a la consulta?

- 3 contestan que sin ninguna preparación.

- 1 en ayunas.

- 1 contesta que en ayunas y con analítica del médico de cabecera.

- Pregunta 12: ¿En qué condiciones debería acudir el enfermo a la consulta?

- 9 en ambas condiciones y 1 en ayunas.

- Pregunta 13: En la misma consulta, ¿se realiza ecografía de abdomen al paciente?

- 4 contestan que sí.

- 1 hospital contesta que no. 
- Pregunta 14: ¿Cree que se debería realizar?

- Todos contestan que sí.

- Pregunta 15: ¿Dispone de sistema informático integrado hospitalario?

- 8 contestan que sí, 2 hospitales contestan que no.

- Pregunta 16: ¿Cree que debería disponer de sistema informático integrado hospitalario?

- Todos sí.

- Pregunta 17: ¿Dispone de una enfermera para esa consulta en exclusiva?

- 3 contestan que no y 2 contestan que sí.

-Pregunta 18: ¿Cree que debería disponer de una enfermera para esa consulta en exclusiva?

- Todos sí.

- Pregunta 19: En general, ¿en cuántas visitas se emite un diagnóstico del paciente?

- 3 contestan en 2, 2 contestan en 1 o en 2 .

- Pregunta 20: En general, ¿en cuántas visitas se debería emitir un diagnóstico del paciente?

- Todos contestan que en 1 ó 2.

- Pregunta 21: ¿Quién vuelve a citar el enfermo?

- 3 contestan el médico, 1 contesta la enfermera y otro el administrativo.

- Pregunta 22: ¿Quién debería volver a citar el enfermo?

- 8 el médico y 2 la enfermera.

- Pregunta 23: En caso de no poderse dar de alta al paciente por presentar una patología que precise revisiones por digestivo...

- 4 contestan que se deriva a otra consulta y 1 que se sigue en esta CARE.

- Pregunta 24: ¿Qué piensa que debería hacerse en el anterior caso expuesto?

- Todos contestan que se deriva a otra consulta

- Pregunta 25: ¿Cree que es útil esta consulta?

- Todos sí.

- Pregunta 26: En caso afirmativo, ¿por qué?

- Mayor colaboración entre Atención Primaria y FEA. Protocolos conjuntos.

- Flexibilidad en las consultas. Filtro adecuado para el resto de las consultas.

- Permite una orientación rápida de la patología del paciente. Integra procesos como colecistitis o dispepsia. Se resuelven patologías concretas.

- Tiene capacidad resolutiva alta. Evita ingresos innecesarios.

- Aúna todos los esfuerzos para minimizar las esperas.

\section{DISCUSIÓN}

En este estudio, similar al recientemente publicado por este grupo sobre las unidades de endoscopia, se trata de analizar cómo está desarrollándose este nuevo sistema de consulta en Andalucía. Se trata de una consulta que trata de atender a los pacientes de una forma rápida y eficaz, aprovechando al máximo los recursos.

Los objetivos de la CARE son:

1. Atender a pacientes nuevos.

2. Servir de filtro para consultas más especialidades dentro del servicio:

- Hepatología.

- Enfermedad inflamatoria intestinal.

- Páncreas y vía biliar.

-ERGE.

3. Emitir un diagnóstico o al menos una orientación diagnóstica, en una o dos visitas del paciente (primera visita y la segunda de recogida de resultados).

En base a estos objetivos se puede definir una CARE como aquel proceso asistencial ambulatorio de fácil acceso para el paciente en el que quede establecido un diagnóstico junto con su tratamiento tras la realización de las pruebas complementarias necesarias, todo ello con un informe y realizado en una o dos jornadas y en un tiempo aceptable.

En cuanto a las preguntas contestadas por los distintos hospitales encuestados hay que destacar que de los 10 hospitales entrevistados sólo 5 tienen consulta de alta resolución, si bien todos consideran que la deberían tener. Parece claro que el número de pacientes atendidos en este tipo de consulta debe ser bajo, unos 10 y en el propio hospital. Sin embargo, las siguientes preguntas plantean diversas respuestas. No se sabe muy bien si los pacientes deben provenir desde urgencias o desde el médico de cabecera. Ello dependerá del objetivo principal de la consulta, si es atender a pacientes urgentes o es atender a cualquier tipo de pacientes, pero sólo en 2 visitas proporcionar un diagnóstico y un tratamiento. Uno de los hospitales contesta que sólo se deben derivar pacientes cuya patología pueda ser diagnosticada mediante ecografía y/o endoscopia.

El paciente, según los encuestados, debería acudir a la consulta en ayunas y con analítica del médico de cabecera, para así poder realizarles la ecografía sobre en la primera visita. Además la consulta debe constar de sistema informático integrado a la red del hospital y de una enfermera propia. Los pacientes deberían ser citados por el propio médico estableciéndose un diagnóstico en la mayor parte de los casos en 1 ó 2 visitas. Por último, según los encuestados este tipo de consultas es muy útil en nuestro actual sistema, porque permite una mayor colaboración entre Atención Primaria y FEA y flexibilidad en las consultas. Permite una orientación rápida de la patología del paciente y actúa de filtro adecuado para el resto de las consultas. Tiene capacidad resolutiva alta, evitando ingresos innecesarios. 


\section{CONCLUSIONES}

En función de las respuestas dadas a las preguntas por los encuestados, se pueden sacar una serie de conclusiones. Creemos que la consulta deber ser atendida por un facultativo especialista de área (FEA) con conocimientos y experiencia en la realización de ecografía y endoscopia digestiva, y en el manejo de programas informáticos a nivel de usuario. La consulta debe constar de: ecógrafo en la consulta, ordenador con programa de gestión de informes y conexión a internet, auxiliar o enfermera de continua presencia en la consulta, al menos durante las horas de visita de los pacientes.

Durante la primera visita se deben realizar las siguientes actuaciones:

- Los pacientes deben acudir a la consulta en ayunas.

- Historia clínica y exploración.

- Ecografía de abdomen.

- Solicitud de analítica (para que se la haga ese mismo día).

- Solicitud de pruebas complementarias.

En la segunda visita o visita de resultados:
- Valoración de las pruebas complementarias.

- Emitir un diagnóstico y poner tratamiento.

- Hacer un informe para el médico de cabecera con las distintas pruebas realizadas, el juicio clínico y el tratamiento a seguir.

- En caso de detectarse alguna patología no subsidiaria de seguimiento por el médico de cabecera se derivará a la consulta especializada.

El tiempo que estimamos entre la primera y segunda visita, va a depender de la patología sospechada, de la urgencia de la derivación y de las pruebas complementarias solicitadas. Por esto, no es recomendable establecer siempre el mismo tiempo.

\section{BIBLIOGRAFÍA}

1. Saldaña ML, Reparaz J, Carnicero J, Asiain J, López G, Lorenzo S. Aplicación del "Appropriateness Evaluation Protocol" en un hospital de agudos. Gestión Hospitalaria 1996; 1: 10-9.

2. Herrerías Gutiérrez JM, Argüelles Arias F; on behalf of Andalusian Team of Chiefs of Gastroenterology Units and Departments. Results of a survey in Andalusian endoscopy units. Rev Esp Enferm Dig 2006; 98 (12): 929-48. 